\title{
Blind Hyperspectral and Multispectral Image Fusion Using Coupled Non-Negative Tucker Tensor Decomposition
}

\author{
Marzieh Zare, Mohammad Sadegh Helfroush, and Kamran Kazemi
}

\begin{abstract}
Fusing a low spatial resolution hyperspectral image (HSI) with a high spatial resolution multispectral image (MSI) to produce a fused high spatio-spectal resolution one, referred to as HSI super-resolution, has recently attracted increasing research interests. In this paper, a new method based on coupled nonnegative tensor decomposition (CNTD) is proposed. The proposed method uses tucker tensor factorization for low resolution hyperspectral image (LR-HSI) and high resolution multispectral image (HR-MSI) under the constraint of non-negative tensor decomposition (NTD). The conventional non-negative matrix factorization (NMF) method essentially loses spatio-spectral joint structure information when stacking a 3D data into a matrix form. On the contrary, in NMF-based methods, the spectral, spatial, or their joint structures must be imposed from outside as a constraint to well pose the NMF problem, The proposed CNTD method blindly brings the advantage of preserving the spatio-spectral joint structure of HSIs. In this paper, the NTD is imposed on the coupled tensor of HSI and MSI straightly. Hence the intrinsic spatio-spectral joint structure of HSI can be losslessly expressed and interdependently exploited. Furthermore, multilinear interactions of different modes of the HSIs can be exactly modeled by means of the core tensor of the Tucker tensor decomposition. The proposed method is completely straightforward and easy to implement. Unlike the other state-of-the-art methods, the complexity of the proposed CNTD method is quite linear with the size of the HSI cube. Compared with the state-of-the-art methods experiments on two well-known datasets, give promising results with lower complexity order.
\end{abstract}

Index Terms - High resolution multispectral image (HR-MSI), Image Fusion, Low resolution hyperspectral image (LR-HSI), Multiplicative update rules (MUR), Non-negative Tucker tensor decomposition.

\section{INTRODUCTION}

$\mathrm{H}$ YPERSPECTRAL imagery is a technique that utilizes a wide range of electromagnetic spectrum in image acquisition in order to yield more information on what is imaged and identify materials or detect processes. As each band of hyperspectral image (HSI) is the spectral response to a narrow band of the electromagnetic spectrum, it is necessary to collect reflections from a wider area on the scene, decreasing the spatial resolution

The authors are with the Department of Electrical and Electronics Engineering, Shiraz University of Technology, Shiraz 715555-313, Iran(e-mail: ma.zare@sutech.ac.ir; ms_helfroush@sutech.ac.ir; kazemi@sutech.ac.ir). of HSIs. There are several attempts aiming to increase the spatial resolution of HSIs in recent years [1-13]. Basically, all super-resolution HSI approaches can be described in three categories; fusing low resolution hyperspectral image (LR-HSI) and high resolution multispectral image (HR-MSI) as a Bayesian framework [3, 11, 14-19], non-negative matrix factorization (NMF) based methods [4, 20-25] and tensor factorization based methods [1, 2, 8, 26-29]. Bayesian frameworks build the posterior distribution based on observed LR-HSI and HR-MSI and some prior information or regularization term and utilize the alternating direction method of multipliers (ADMM) [11] optimization method to estimate super-resolution HSI. In [11] a Bayesian framework based on a sparse representation is introduced to solve the fusion problem, thus, the abundance fractions are estimated using ADMM. In our previous work [30], a smooth graph signal modeling in a Bayesian framework is employed to impose the consistency of subspace projection fractions corresponding to the nearby nodes by exploiting the graph Laplacian matrix. The method proposed in [31] exploits non-local self-similarity based on a spatial and spectral sparse representation to estimate both matrices of abundance fractions and endmembers, which the product of these two matrices leads to the super-resolution HSI. The method proposed in [5] uses spectral unmixing and Bayesian sparse representation to enhance the spatial resolution of hyperspectral images. The most important deficiency of the Bayesian frameworks is the necessity to the regularization terms. They should be able to comprehensively represent spatial information of HSIs, which is lost in the HSI matricizing of HSIs stage.

As an alternative approach, non-negative matrix factorization (NMF) has been widely proposed to fuse pairs of LR-HSIs and HR-MSIs. This methods brings the advantages of clear physical, statistical, and geometric inference, flexible modeling, and less requirement on prior information [32]. As HSIs are essentially non-negative data, NMF frameworks are quite compatible with the observed data. These approaches first unfold the HSI into the matrix form and factorize it into two non-negative matrices, called as the basis and the coefficient matrices. In order to avoid trapping in the local minima due to its non-convex objective function, several constraint NMF methods $[4,22,23,33]$ are introduced. Instead of the original $\mathrm{L}_{1}$ regularizer, the work in [22] utilizes $\mathrm{L}_{1 / 2}$ regularizer, on the 
abundance fractions to enforce the sparse estimation. In [23] a multilayer NMF spectral unmixing is introduced, where the products of the sub-layer output factors lead to the superresolution image. In [33], using sparsity-constrained deep NMF with the total variation regularization, to estimate HR-HSI.

Noteworthy, stacking a 3D data into matrix form in NMFbased approaches loses the neighborhood structures, smoothness, and continuity characteristics. In this regard, tensors or multiway arrays have been frequently used in multidimensional data analysis [26, 28, 34-36]. Additionally, exploiting the power of multilinear algebra of the tensor representation shows more flexibility in the choice of constraints that match data properties. It also can extract more general latent components in the data compared with the matrix-based approaches. More recently tensor-based representation of HSI is widely being used [1, 8, 26, 28, 32]. Representation of an HSI as a third order tensor is a structural and natural without any information loss model. In most of these methods, a low rank tensor representation is exploited to estimate the original HR-HSI. It offers the benefit of extremely noise and memory usage reduction, and extracting discriminative features[32]. Accordingly, two well-known tensor decomposition, Tucker decomposition and canonical polyadic decomposition (CPD) are widely used to approximate HR-HSIs [8, 26, 28, 29, 37, 38]. Learning a low tensor-train rank representation is incorporated in [26] for hyperspectral image super-resolution. In [28] a nonlocal coupled canonical polyadic (CP) tensor decomposition framework is used to fuse hyperspectral and multispectral images. The spatio-spectral sparsity prior of HSIs is the constraint that is imposed on the core tensor in the CSTF method [8]. Also in [27], nonlocal sparse tensor factorization (NLSTF) of HSIs is proposed to model non-local self-similarity of HSIs.

As a strong prior knowledge, HSIs are naturally non-negative data. Thus, applying non-negative constraints to the tensor factorization method is expected to further improve the performance of the fusion method. In this paper, we extend NMF to a tensor framework which is called non-negative tensor decomposition (NTD). Contrary to the conventional NMFbased methods, where, the spectral, spatial, or their joint structures must be additionally imposed as a constraint, in this paper, a multiway representation of HSIs preserves the spatiospectral joint structures of HSIs with no prior knowledge resulting a blind framework. We take spectral advantages of LR-HSI and spatial advantages of HR-MSI, via straightly applying coupled Tucker decomposition to both of the above images. Therefore, the proposed method is called CNTD. The proposed method comprehensively models multilinear modes interactions of HSIs, using Tucker tensor representation, where the core tensor precisely expresses relations among different modes. The proposed algorithm is straightforward and easy to implement, where, the complexity is quite linear with the size of the hyperspectral data cube. The main contributions of this paper are highlighted below.

- Extending NMF to a tensor framework, which is called NTD.
- Applying coupled Tucker decomposition to the LR-HSI and HR-MSI, to estimate spectral and spatial information of HR-HSI, respectively.

- $\quad$ Preserving spatio-spectral joint structures of HSIs with no information loss and no prior knowledge requirement, using a multiway representation of HSIs.

- Giving Lower complexity order comparing with the other state-of-the-art methods.

The remainder of this paper is organized as follows. Some preliminaries on tensors are presented in Section II. Section III formulates the HSI-MSI fusion. The proposed coupled nonnegative tensor decomposition (CNTD) method for blind superresolution HSI is introduced in Section IV. The complexity order of the proposed method is mentioned in Section V. The experimental results on two well-known datasets Pavia University and Indian Pines, are illustrated in Section VI. Finally, conclusion and our future work are given in Section VII.

\section{PRELIMINARIES ON TENSORS}

An $N$-dimensional tensor $\mathcal{X} \in \mathbb{R}^{I_{1} \times I_{2} \times \ldots \times I_{N}}$ has $N$ indices $i_{1}, i_{2}, \ldots, i_{N}$ and its elements are denoted by $x_{i_{1} i_{2} \ldots i_{N}}$ where $1 \leq$ $i_{n} \leq I_{n}$. Tensor matricization unfolds an $N$-dimensional tensor into a matrix. The mode- $n$ matricization of $\mathcal{X}$ reorders/unfolds the elements of $\mathcal{X}$ to form the matrix $\mathbf{x}_{(n)} \in$ $\mathbb{R}^{I_{n} \times I_{n+1} I_{n+2} \ldots I_{N} I_{1} I_{2} \ldots I_{n-1}}$. Noteworthy that, matricization of a tensor is quite analogous to matrix vectorization. The mode- $n$ product of the tensor $\mathcal{X} \in \mathbb{R}^{I_{1} \times I_{2} \times \ldots \times I_{N}}$ by the matrix $\mathbf{A} \in$ $\mathbb{R}^{I_{n} \times I_{n}}$, is defined by $\mathcal{X} \times{ }_{n} \mathbf{A}$, is an $N$-dimensional tensor $\mathcal{M} \in$ $\mathbb{R}^{I_{1} \times I_{2} \times \ldots \times J_{n} \times \ldots \times I_{N}}$, which entries are calculated by

$$
m_{i_{1} \ldots i_{n-1} J_{n} i_{n+1} \ldots i_{N}}=\sum_{i_{n}} x_{i_{1} \ldots i_{n-1} i_{n} i_{n+1} \ldots i_{N}} a_{j_{n} i_{n}}
$$

The mode- $n$ product $\boldsymbol{X} \times_{n}$ A can also be calculated in matrix form as $\mathbf{M}_{(n)}=\mathbf{A} \mathbf{X}_{(n)}$. For multiple mode- $n$ product the order is irrelevant, which is

$$
\mathcal{X} \times_{m} \mathbf{A} \times_{n} \mathbf{B}=\mathcal{X} \times_{n} \mathbf{B} \times_{m} \mathbf{A} \quad(m \neq n)
$$

and for multiple mode- $n$ products with the same modes, the order is relevant, which is

$$
\mathcal{X} \times_{n} \mathbf{A} \times_{n} \mathbf{B}=\mathcal{X} \times_{n}(\mathbf{B A})
$$

The scalar product of two tensors $\mathcal{X}, \mathcal{Y}$ indicated as $\langle\boldsymbol{X}, \boldsymbol{y}\rangle$ $=\sum_{i_{1}, i_{2}, \ldots, i_{N}} x_{i_{1}, i_{2}, \ldots, i_{N}} \mathcal{Y}_{i_{1}, i_{2}, \ldots, i_{N}}$. The Frobenius norm of a tensor $\boldsymbol{X}$ is indicated as $\|\boldsymbol{X}\|_{F}=\sqrt{\langle\boldsymbol{X}, \boldsymbol{X}\rangle}$.

The mode- $n$ product has two important properties as follows

$$
\left(\boldsymbol{X} \times_{n} \mathbf{A}\right) \times_{m} \mathbf{B}=\left(\boldsymbol{X} \times_{m} \mathbf{B}\right) \times_{n} \mathbf{A}
$$

$$
\left(\boldsymbol{X} \times_{n} \mathbf{A}\right) \times_{n} \mathbf{B}=\mathcal{X} \times_{n}(\mathbf{B A})
$$


TABLE I

BASIC NOTATION

\begin{tabular}{cc}
\hline \hline Notation & Description \\
\hline $\boldsymbol{X}$ & Tensor \\
$\mathbf{X}$ & Matrix \\
$x$ & Tensor element \\
$\boldsymbol{x}$ & Spectral vector of tensor \\
$x$ & Scaler \\
$\times_{n}$ & Mode- $n$ product \\
$\otimes$ & Kronecker product \\
$\circledast$ & Hadamard product \\
$\mathbf{X}_{(n)}$ & Mode- $n$ matricization of tensor $\mathrm{X}$ \\
$\mathbf{X}^{(n)}$ & $n$ mode matrix in Tucker model \\
\hline \hline
\end{tabular}

The Tucker decomposition of the $N$-dimensional tensor $\boldsymbol{X} \in$ $\mathbb{R}^{I_{1} \times I_{2} \times \ldots \times I_{N}}$ is expressed as mode products of a core tensor $\mathcal{U} \in$ $\mathbb{R}^{K_{1} \times K_{2} \times \ldots \times K_{N}}$ and $N$ mode matrices $\mathbf{V}^{(\boldsymbol{n})} \in \mathbb{R}^{I_{n} \times K_{n}}$, which is expressed as

$$
\mathcal{X}=\mathcal{U} \times_{1} \mathbf{V}^{(1)} \times_{2} \mathbf{V}^{(2)} \ldots \times_{N} \mathbf{V}^{(N)}
$$

which element-wise form is as below

$$
x_{i_{1} \ldots i_{N}}=\sum_{k_{1} \ldots k_{N}} u_{k_{1} \ldots k_{N}} v_{i_{1} k_{1}}^{(1)} v_{i_{2} k_{2}}^{(2)} \ldots v_{i_{N} k_{N}}^{(N)}
$$

The mode- $n$ matricization form of , is expressed by Kronecker products $(\otimes)$ of the mode- $n$ matricization of the core tensor and mode matrices as follows

$$
\begin{gathered}
\mathbf{X}_{(n)}=\mathbf{V}^{(n)} \mathbf{U}_{(n)}\left[\mathbf{V}^{(\boldsymbol{n}-1)} \otimes \ldots \otimes \mathbf{V}^{(2)} \otimes \mathbf{V}^{(1)} \otimes \mathbf{V}^{(N)}\right. \\
\otimes \ldots \otimes \mathbf{V}^{(n+2)} \otimes \mathbf{V}^{(n+1)}
\end{gathered}
$$

where $\mathbf{U}_{(n)}$ is the mode- $n$ matricization of the core tensor $\mathcal{U}$. The Kronecker product of two matrices $\mathbf{A} \in \mathbb{R}^{I \times J}$ and $\mathbf{B} \in$ $\mathbb{R}^{K \times L}$ is a matrix denoted as $\mathbf{A} \otimes \mathbf{B} \in \mathbb{R}^{I K \times J L}$ is defined as

$$
\mathrm{A} \otimes \mathrm{B}=\left[\begin{array}{cccc}
a_{11} \mathrm{~B} & a_{12} \mathrm{~B} & \cdots & a_{1 J} \mathrm{~B} \\
a_{21} \mathrm{~B} & a_{22} \mathrm{~B} & \cdots & a_{2 J} \mathrm{~B} \\
\vdots & \vdots & \ddots & \vdots \\
a_{I 1} \mathrm{~B} & a_{I 2} \mathrm{~B} & \cdots & a_{I J} \mathrm{~B}
\end{array}\right]
$$

The other properties of Kronecker product and vectorization operation $(\operatorname{vec}(\cdot))$ that are used in this paper are as below

$$
\begin{gathered}
\operatorname{vec}\left(\mathbf{U A \mathbf { V } ^ { T }}\right)=(\mathbf{V} \otimes \mathbf{U}) \operatorname{vec}(\mathbf{A}) \\
(\mathbf{V} \otimes \mathbf{U})^{T}=\mathbf{V}^{T} \otimes \mathbf{U}^{T} \\
(\mathbf{V} \otimes \mathbf{U})(\mathbf{A} \otimes \mathbf{B})=\mathbf{V A} \otimes \mathbf{U B}
\end{gathered}
$$

All basic notations are represented in Table. I.

\section{PROBLEM Formulation}

As HSIs are naturally a 3D data, the tensor is a more efficient representation than matrix form, and we can benefit from its ability to exploit intrinsic structures of HSI and multilinear interactions of its different modes. Hence in this paper, the target HR-HSI is denoted as a three-dimensional tensor $\boldsymbol{Z} \in$ $\mathbb{R}^{W \times H \times S}$, where $W, H$ and $S$ are the dimensions of the width, height and spectral (depth) modes, respectively. It is formally expressed as

$$
\boldsymbol{Z}=\boldsymbol{C} \times_{1} \mathbf{W} \times_{2} \mathbf{H} \times_{3} \mathbf{S}
$$

which is called Tucker representation, where $\mathbf{W} \in \mathbb{R}^{W \times n_{w}}, \mathbf{H} \in$ $\mathbb{R}^{H \times n_{h}}$ and $\mathbf{S} \in \mathbb{R}^{S \times n_{s}}$ are width, height and spectral dictionary matrices, respectively. $n_{w}, n_{h}$ and $n_{s}$ are the number of each mode dictionaries atoms, and $\mathcal{C} \in \mathbb{R}^{n_{w} \times n_{h} \times n_{s}}$ is the core tensor that shows the interactions among different modes.

Mode- $n(n=1,2,3)$ matricization of $\boldsymbol{Z}$ are as follows

$$
\begin{aligned}
& \mathbf{Z}_{(1)}=\mathbf{W C}_{(1)}(\mathbf{S} \otimes \mathbf{H})^{T} \\
& \mathbf{Z}_{(2)}=\mathbf{H C}_{(2)}(\mathbf{S} \otimes \mathbf{W})^{T} \\
& \mathbf{Z}_{(3)}=\mathbf{S C}_{(3)}(\mathbf{H} \otimes \mathbf{W})^{T}
\end{aligned}
$$

where $(\cdot)^{T}$ denotes the transposition of the matrix.

Both LR-HSI and HR-MSI are also denoted as threedimensional tensors $\boldsymbol{y}_{h} \in \mathbb{R}^{w \times h \times S}$ and $\boldsymbol{y}_{m} \in \mathbb{R}^{W \times H \times s}$, respectively. $w, h$ and $s$ are the indexes of the width, height and spectral modes, respectively. LR-HSI and HR-MSI are expressed as below

$$
\begin{aligned}
& \boldsymbol{y}_{h}=\mathcal{C} \times_{1} \mathbf{W}_{\boldsymbol{h}} \times_{2} \mathbf{H}_{\boldsymbol{h}} \times_{3} \mathbf{S}+\mathcal{E}_{\boldsymbol{h}} \\
& \boldsymbol{y}_{m}=\mathcal{C} \times_{1} \mathbf{W} \times_{2} \mathbf{H} \times_{3} \mathbf{S}_{\boldsymbol{m}}+\mathcal{E}_{\boldsymbol{m}}
\end{aligned}
$$

where $\mathbf{W}_{\boldsymbol{h}} \in \mathbb{R}^{\boldsymbol{w} \times \boldsymbol{n}_{\boldsymbol{w}}}, \mathbf{H}_{\boldsymbol{h}} \in \mathbb{R}^{\boldsymbol{h} \times \boldsymbol{n}_{\boldsymbol{h}}}$ and $\boldsymbol{S}_{\boldsymbol{m}} \in \mathbb{R}^{\boldsymbol{s} \times \boldsymbol{n}_{\boldsymbol{s}}}$ are width, height, and spectral dictionary matrices, respectively, and $\mathcal{E}_{\boldsymbol{h}} \in$ $\mathbb{R}^{w \times h \times S}$ and $\mathcal{E}_{\boldsymbol{m}} \in \mathbb{R}^{W \times H \times S}$ are the independent and identically distributed (i.i.d.) noise of $\mathcal{Y}_{h}$ and $\mathcal{Y}_{m}$, respectively.

Conventionally, LR-HSI and HR-HSI are considered to be the spatial and spectral down-sampled version of HR-HSI, respectively. Therefore, the LR-HSI acquisition process can be formulated as

$$
\begin{aligned}
& \mathbf{W}_{h}=\mathbf{P}_{1} \mathbf{W} \\
& \mathbf{H}_{h}=\mathbf{P}_{2} \mathbf{H}
\end{aligned}
$$$$
\boldsymbol{y}_{h}=\mathcal{C} \times_{1}\left(\mathbf{P}_{1} \mathbf{W}\right) \times_{2}\left(\mathbf{P}_{2} \mathbf{H}\right) \times_{3} \mathbf{S}+\mathcal{E}_{\boldsymbol{h}}
$$

where $\mathbf{P}_{1} \in \mathbb{R}^{\boldsymbol{w} \times W}$ and $\mathbf{P}_{2} \in \mathbb{R}^{\boldsymbol{h} \times H}$ are spatial separable downsampling operators of width and height modes, respectively. They are point spread functions (PSF) of imaging sensor and assumed to be known. Similarly, HR-MSI can be expressed as

$$
\boldsymbol{y}_{m}=\mathcal{C} \times_{1} \mathbf{W} \times_{2} \mathbf{H} \times_{3}\left(\mathbf{P}_{3} \mathbf{S}\right)+\mathcal{E}_{\boldsymbol{m}}
$$




$$
\mathbf{S}_{m}=\mathbf{P}_{3} \mathbf{S}
$$

where $\mathbf{P}_{3} \in \mathbb{R}^{s \times S}$ is spectral down-sampling operator, which is spectral response function (SRF) of the imaging sensor and assumed to be known.

\section{PROPOSED CNTD APPROACH FORMULATION}

The goal of fusing LR-HSI and HR-MSI is to estimate a high spatio-spectral target image of HSI. Since $w \ll W, h \ll H$ and $s \ll S$, the super-resolution problem is severely ill-posed, some prior information is needed to regularize the fusion problem. Orthogonality and statistical independency of basis vectors in the Tucker representation, sparsity, smoothness, and nonnegativity of HSIs are some constraints that help to find a unique solution for the super-resolution problem of HSIs [3941].

Accordingly, in this paper, we propose a new method based on coupled non-negative tensor decomposition (CNTD). The proposed method performs Tucker tensor factorization for LRHSI and HR-MSI subject to non-negative tensor decomposition (NTD). The original NMF method inherently loses spatiospectral joint structure information when unfolding a 3D data into the matrix form. Therefore in this paper, we impose NTD to the both tensor of HSI and MSI straightly. The CNTD method effectively combines multiple data tensors, where the intrinsic spatio-spectral joint structures of HSI can be losslessly represented and interdependently exploited. The proposed CNTD method is illustrated in Fig. 1.

Considering (11), (13), and (14), LR-HSI and HR-MSI fusion problem is formulated as the constrained least squares optimization problems, expressed as follows

$$
\min _{\mathcal{C}, \mathbf{w}_{h}, \mathbf{H}_{h}, \mathbf{s}}\left\|\boldsymbol{y}_{h}-\mathcal{C} \times{ }_{1} \mathbf{W}_{h} \times_{2} \mathbf{H}_{h} \times_{3} \mathbf{S}\right\|_{F}^{2}
$$

$$
\text { s.t. } \mathcal{C}, \mathbf{W}_{h}, \mathbf{H}_{h}, \mathbf{S} \geq 0
$$

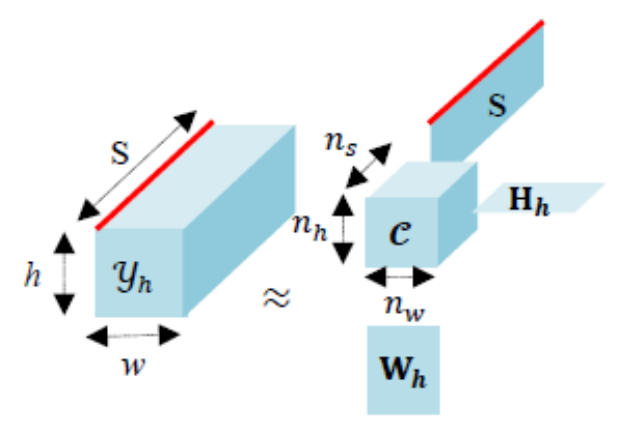

(a)

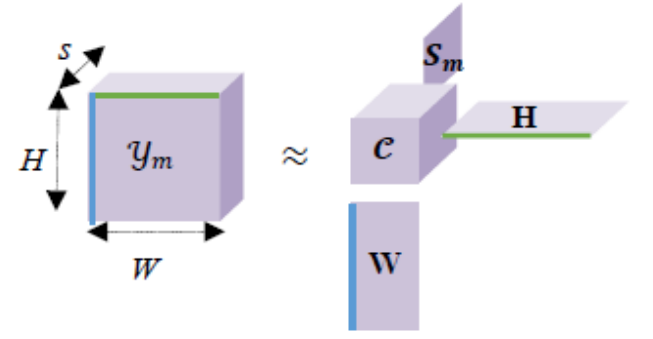

(b)

Fig. 1. Illustration of the proposed CNTD method for hyperspectral and multispectral data fusion; (a) the LR-HSI Tucker decomposition, (b) the HR-MSI Tucker decomposition, (c) the fused superresolution image (HR-HSI).

$$
\min _{\mathcal{C}, \mathbf{W}, \mathbf{H}, \mathbf{s}_{\boldsymbol{m}}}\left\|\boldsymbol{y}_{m}-\mathcal{C} \times_{1} \mathbf{W} \times_{2} \mathbf{H} \times_{3} \mathbf{S}_{\boldsymbol{m}}\right\|_{F}^{2}
$$

$$
\text { s.t. } \mathcal{C}, \mathbf{W}_{h}, \mathbf{H}_{h}, \mathbf{S} \geq 0
$$

where $\|\cdot\|_{F}$ denotes the Frobenius norm. Hyperspectral and multispectral data fusion based on non-negative Tucker decomposition are achieved by the estimation of the corresponding dictionaries and the core tensor. NTD is straightforward to formulate and easy to implement likewise the conventional NMF. Non-negative Tucker tensor decomposition attempts to decompose a non-negative data tensor into the multilinear products of a non-negative core tensor and nonnegative mode dictionary matrices [42]. We execute the multiplicative update rule (MUR) to minimize the predefined optimization problems (20) and (21). We perform the multiplicative updating algorithms for NTD, which we directly derive from NMF multiplicative algorithms. The convergence to local optima under the non-negativity constraints has been proven in [20,43], which can be applied to our case as well.

\section{A. Updating mode dictionary matrices}

Updating algorithms for each mode dictionary matrices can be easily derived by matricizing the Tucker model into associated modes. We use extended MUR for the NTDs of $\boldsymbol{y}_{h}$, hence the first mode matricization of $\boldsymbol{y}_{h}$ is as below

$$
\mathbf{Y}_{\boldsymbol{h}_{(1)}} \approx \underbrace{\mathbf{W}_{\boldsymbol{h}}}_{\begin{array}{c}
\text { 1st fraction of the } \\
\text { conventional NMF }
\end{array}} \underbrace{\mathbf{C}_{(1)}\left(\mathbf{S} \otimes \mathbf{H}_{\boldsymbol{h}}\right)^{\boldsymbol{T}}}_{\begin{array}{c}
\text { nd fraction of the } \\
\text { contional NMF }
\end{array}}
$$

where $\mathbf{Y}_{\boldsymbol{h}_{(1)}}$ and $\mathbf{C}_{(1)}$ are the first mode matricization of LRHSI $\left(\boldsymbol{y}_{h}\right)$ and the core tensor $(\boldsymbol{C})$, respectively. Equation (22) can be treated as the conventional NMF, where each fraction is updated using MUR.

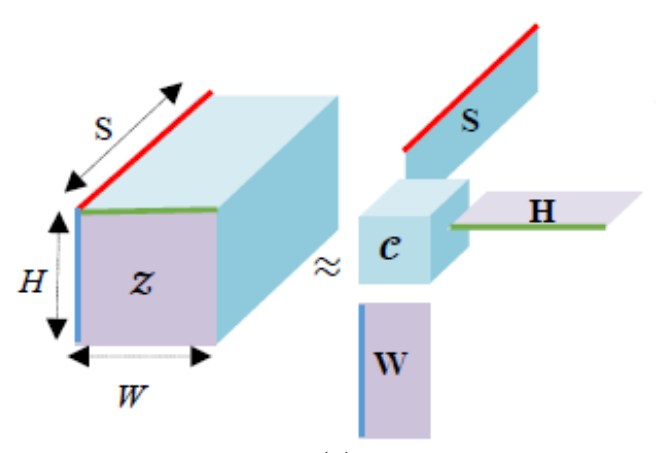

(c) 
Considering (22), the first mode dictionary is updated as

$$
\mathbf{W}_{h} \leftarrow \mathbf{W}_{h} \circledast \frac{\mathbf{Y}_{h_{(1)}}\left[\mathrm{C}_{(1)}\left(S \otimes \mathbf{H}_{h}\right)^{T}\right]^{T}}{\mathbf{W}_{h} \mathbf{C}_{(1)}\left(S \otimes H_{h}\right)^{T}\left[\mathrm{C}_{(1)}\left(S \otimes H_{h}\right)^{T}\right]^{T}}
$$

where the fraction line is used here to denote the element-wise division.

Analogously the second mode dictionary matrix can be updated using the second mode matricization of $\boldsymbol{y}_{h}$, which is as

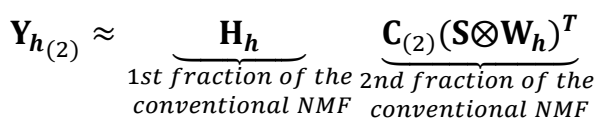

and the second mode dictionary matrix is updated as below

$$
\mathbf{H}_{h} \leftarrow \mathbf{H}_{h} \circledast \frac{\mathbf{Y}_{h_{(2)}}\left[\mathrm{C}_{(2)}\left(S \otimes W_{h}\right)^{T}\right]^{T}}{\mathbf{H}_{h} \mathbf{C}_{(2)}\left(S \otimes W_{h}\right)^{T}\left[\mathrm{C}_{(2)}\left(S \otimes W_{h}\right)^{T}\right]^{T}}
$$

Equivalently the third mode matricization of $\boldsymbol{y}_{h}$ is $\mathbf{Y}_{\boldsymbol{h}_{(2)}} \approx$ $\mathbf{S C}_{(3)}\left(\mathbf{H}_{\boldsymbol{h}} \otimes \mathbf{W}_{\boldsymbol{h}}\right)^{\boldsymbol{T}}$. Therefore, the spectral mode dictionary (S) is updated as

$$
\mathrm{S} \leftarrow \mathrm{S} \circledast \frac{\mathbf{Y}_{h_{(3)}}\left[\mathrm{C}_{(3)}\left(\mathbf{H}_{h} \otimes \mathbf{W}_{h}\right)^{T}\right]^{T}}{\mathrm{SC}_{(3)}\left(\mathbf{H}_{h} \otimes \mathbf{W}_{h}\right)^{T}\left[\mathrm{C}_{(3)}\left(\mathbf{H}_{h} \otimes \mathbf{W}_{h}\right)^{T}\right]^{T}}
$$

\section{B. Updating core tensor}

Considering (10) and (13) we have

$$
\begin{aligned}
\operatorname{vec}\left(\mathbf{Y}_{\boldsymbol{h}_{(1)}}\right) & =\operatorname{vec}\left(\mathbf{W}_{\boldsymbol{h}} \mathbf{C}_{(1)}\left(\mathbf{S} \otimes \mathbf{H}_{\boldsymbol{h}}\right)^{\boldsymbol{T}}\right) \\
= & \underbrace{\operatorname{vec}\left(\mathbf{C}_{(1)}\right)}_{\begin{array}{c}
\text { 1st fraction of the } \\
\text { conventional NMF }
\end{array} \underbrace{\left(\mathbf{S} \otimes \mathbf{H}_{\boldsymbol{h}} \otimes \mathbf{W}_{\boldsymbol{h}}\right)}_{\text {conventional NMF }}}
\end{aligned}
$$

which can be treated as the conventional NMF as well. Incorporating MUR to calculate the core tensor $(\boldsymbol{C})$, which is as

$$
\begin{aligned}
& \operatorname{vec}\left(\mathbf{C}_{(1)}\right) \leftarrow \operatorname{vec}\left(\mathbf{C}_{(1)}\right) \\
& \circledast \frac{\left(\mathbf{S} \otimes \mathbf{H}_{h} \otimes \mathbf{W}_{\boldsymbol{h}}\right)^{T} \operatorname{vec}\left(\mathbf{Y}_{\boldsymbol{h}_{(1)}}\right)}{\left(\mathbf{S} \otimes \mathbf{H}_{\boldsymbol{h}} \otimes \mathbf{W}_{\boldsymbol{h}}\right)^{\boldsymbol{T}}\left(\mathbf{S} \otimes \mathbf{H}_{\boldsymbol{h}} \otimes \mathbf{W}_{\boldsymbol{h}}\right) \operatorname{vec}\left(\mathbf{C}_{(1)}\right)}
\end{aligned}
$$

considering (10) and (22) the numerator of (28) is as

$$
\begin{aligned}
\left(\mathbf{S} \otimes \mathbf{H}_{h}\right. & \left.\otimes \mathbf{W}_{h}\right)^{T} \operatorname{vec}\left(\mathbf{Y}_{\boldsymbol{h}_{(1)}}\right) \\
& =\left(\left(\mathbf{S} \otimes \mathbf{H}_{h}\right)^{T} \otimes \mathbf{W}_{h}{ }^{T}\right) \operatorname{vec}\left(\mathbf{Y}_{\boldsymbol{h}_{(1)}}\right) \\
& =\operatorname{vec}\left(\mathbf{W}_{\boldsymbol{h}}{ }^{T} \mathbf{Y}_{\boldsymbol{h}_{(1)}}\left(\mathbf{S}^{T} \otimes \mathbf{H}_{h}{ }^{T}\right)^{T}\right) \\
& =\operatorname{vec}\left(\left(\boldsymbol{y}_{h} \times_{1} \mathbf{W}_{h}{ }^{T} \times_{2} \mathbf{H}_{h}{ }^{T} \times_{3} \mathbf{S}^{T}\right)_{(1)}\right)
\end{aligned}
$$

and its denominator considering (10) is as

$$
\begin{aligned}
& \left(\mathbf{S} \otimes \mathbf{H}_{h} \otimes \mathbf{W}_{h}\right)^{T}\left(\mathbf{S} \otimes \mathbf{H}_{h} \otimes \mathbf{W}_{h}\right) \operatorname{vec}\left(\mathbf{C}_{(1)}\right) \\
& =\left(\left(\mathbf{S} \otimes \mathbf{H}_{\boldsymbol{h}}\right)^{\boldsymbol{T}} \otimes \mathbf{W}_{\boldsymbol{h}}{ }^{\boldsymbol{T}}\right)\left(\left(\mathbf{S} \otimes \mathbf{H}_{\boldsymbol{h}}\right) \otimes \mathbf{W}_{\boldsymbol{h}}\right) \operatorname{vec}\left(\mathbf{C}_{(1)}\right) \\
& =\left[\left(\left(\mathbf{S} \otimes \mathbf{H}_{h}\right)^{T}\left(\mathbf{S} \otimes \mathbf{H}_{h}\right)\right) \otimes\left(\mathbf{W}_{h}{ }^{T} \mathbf{W}_{h}\right)\right] \operatorname{vec}\left(\mathbf{C}_{(1)}\right) \\
& =\operatorname{vec}\left(\left(\mathbf{W}_{\boldsymbol{h}}{ }^{T} \mathbf{W}_{\boldsymbol{h}}\right) \mathbf{C}_{(1)}\left(\mathbf{S}^{\mathrm{T}} \mathbf{S} \otimes \boldsymbol{H}_{\boldsymbol{h}}{ }^{T} \boldsymbol{H}_{\boldsymbol{h}}\right)\right) \\
& =\operatorname{vec}\left(\left(\boldsymbol{C} \times{ }_{1} \mathbf{W}_{h}^{T} \mathbf{W}_{h} \times_{2} \mathbf{H}_{h}^{T} \mathbf{H}_{h} \times_{3} \mathbf{S}^{T} \mathbf{S}\right)_{(1)}\right)
\end{aligned}
$$

As a result the core tensor $\mathcal{C}$ is updated as

$$
\mathcal{C} \leftarrow \mathcal{C} \circledast \frac{\boldsymbol{y}_{h} \times{ }_{1} \mathbf{W}_{h}^{T} \times{ }_{2} \mathbf{H}_{h}^{T} \times{ }_{3} \mathbf{S}^{T}}{\mathcal{C} \times_{1} \mathbf{W}_{h}^{T} \mathbf{W}_{h} \times{ }_{2} \mathbf{H}_{h}^{T} \mathbf{H}_{h} \times{ }_{3} \mathbf{S}^{T} \mathbf{S}}
$$

We also perform MUR for $\mathcal{y}_{m}$ in a similar way, which is detailed for $\mathcal{Y}_{h}$. Accordingly updating relations for $\mathcal{Y}_{m}$ factors are given as follows

$$
\begin{aligned}
& \mathbf{W} \leftarrow \mathbf{W} \circledast \frac{\mathbf{Y}_{m_{(1)}}\left[\mathbf{C}_{(1)}\left(\mathbf{S}_{\boldsymbol{m}} \otimes H\right)^{T}\right]^{T}}{\mathbf{W C}_{(1)}\left(\mathbf{S}_{\boldsymbol{m}} \otimes H\right)^{T}\left[\mathbf{C}_{(1)}\left(\mathbf{S}_{\boldsymbol{m}} \otimes \mathbf{H}\right)^{T}\right]^{T}} \\
& \mathbf{H} \leftarrow \mathbf{H} \circledast \frac{\mathbf{Y}_{m_{(2)}}\left[\mathbf{C}_{(2)}\left(\mathbf{S}_{\boldsymbol{m}} \otimes \mathbf{W}\right)^{T}\right]^{T}}{\mathbf{H C}_{(2)}\left(\mathbf{S}_{\boldsymbol{m}} \otimes \mathbf{W}\right)^{T}\left[\mathbf{C}_{(2)}\left(\mathbf{S}_{\boldsymbol{m}} \otimes \mathbf{W}\right)^{T}\right]^{T}}
\end{aligned}
$$

$$
\begin{gathered}
\mathbf{S}_{\boldsymbol{m}} \leftarrow \mathrm{S}_{\boldsymbol{m}} \circledast \frac{\mathbf{Y}_{\boldsymbol{m}_{(3)}}\left[\mathbf{C}_{(3)}(\mathbf{H} \otimes \mathbf{W})^{T}\right]^{T}}{\mathbf{S}_{\boldsymbol{m}} \mathbf{C}_{(3)}(\mathbf{H} \otimes \mathbf{W})^{T}\left[\mathbf{C}_{(3)}(H \otimes W)^{T}\right]^{T}} \\
\mathcal{C} \leftarrow \mathcal{C} \circledast \frac{\boldsymbol{y}_{m} \times{ }_{1} \mathbf{W}^{T} \times{ }_{2} \mathbf{H}^{T} \times{ }_{3} \mathbf{S}_{\boldsymbol{m}}{ }^{T}}{\mathcal{C} \times_{1} \times{ }_{1} \mathbf{W}^{T} \mathbf{W} \times{ }_{2} \mathbf{H}^{T} \mathbf{H} \times{ }_{3} \mathbf{S}_{\boldsymbol{m}}{ }^{T} \mathbf{S}_{\boldsymbol{m}}}
\end{gathered}
$$

The CNTD proposed algorithm starts from NTD for the LRHSI owing to its spectral information. As the initialization phase, we use the method used in [8]. $\mathbf{W}_{h}, \mathbf{H}_{h}, \mathbf{S}$ and $\mathcal{C}$ via (16), (17), (26), and (31), respectively while the other variables are fixed to inherit the reliable spatial information obtained from multispectral data. Then, $\mathbf{W}_{\boldsymbol{h}}, \mathbf{H}_{\boldsymbol{h}}, \mathbf{S}$ and $\boldsymbol{C}$ are alternately updated via (23), (25), (26), and (31), respectively until convergence of the cost function in (20).

The next step of the proposed algorithm is applying NTD to the HR-MSI. As the initialization phase, $\mathbf{S}_{m}$ is set by (19) and $\mathbf{W}, \mathbf{H}$ and $\boldsymbol{C}$ are updated using (32), (33), and (35), respectively benefiting the spectral information of LR-HSI. As the optimization phase, $\mathbf{W}, \mathbf{H}, \mathbf{S}_{\boldsymbol{m}}$ and $\boldsymbol{C}$ are alternately updated by (32)-(35) while the other variables are fixed, until convergence of the cost function in (21). The superresolution HSI is calculated using the estimated core tensor and mode dictionary matrices. Table. II illustrates the procedure of the proposed CNTD method. 
TABLE II

Algorithm. 1 THE Proposed COUPLED NON-NEGATIVE TENSOR DECOMPOSITION BASED METHOD PROCEDURE

\section{Input: LR-HSI $\left(\boldsymbol{y}_{h}\right)$, HR-MSI $\left(\boldsymbol{y}_{m}\right)$.}

Output: HR-HSI $(\boldsymbol{Z})$

1. Estimate PSF $\left(\mathbf{P}_{1}, \mathbf{P}_{2}\right)$, SRF $\left(\mathbf{P}_{3}\right)$, using method presented in [44].

2. Initialize the core tensor $(\boldsymbol{C})$ and mode dictionaries $(\mathbf{W}, \mathbf{H}, \mathbf{S})$ using method presented in [8].

3. NTD for $\boldsymbol{y}_{h}$ Initialize $\mathbf{W}_{h}, \mathbf{H}_{h}, \mathbf{S}$ and $\mathcal{C}$ via (16), (17), (26) and (31), respectively.

Update $\mathbf{W}_{\boldsymbol{h}}, \mathbf{H}_{\boldsymbol{h}}, \mathbf{S}$ and $\mathcal{C}$ alternately via (23), (25), (26) and (31), respectively until convergence of the cost function in (20).

4. NTD for $\boldsymbol{y}_{m}$

Initialize $\mathbf{S}_{m}, \mathbf{W}, \mathbf{H}$ and $\boldsymbol{C}$ via (19), (32) and (33), (35) respectively.

Update $\mathbf{W}, \mathbf{H}, \mathbf{S}_{\boldsymbol{m}}$ and $\boldsymbol{C}$ alternately via (32)-(35) until convergence of the cost function in (21).

5. Using the estimated $\mathbf{W}, \mathbf{H}, \mathbf{S}$ and $\boldsymbol{C}$ to calculate the HRHSI $(\boldsymbol{Z})$ via Tucker tensor decomposition, which is represented in (11).

\section{COMPUTATIONAL COMPLEXITY}

Finally, we analyze the computational complexity of the proposed method. According to Algorithm 1, the proposed method includes two sub-optimization problems, which engage MURs to estimate $\boldsymbol{y}_{h}$ and $\boldsymbol{y}_{m}$ factors. Each sub-optimization problem mainly contains four updating steps. Therefore, the overall computational complexity of the proposed algorithm is briefly expressed as

$$
\begin{aligned}
& \boldsymbol{O}\left(n_{\boldsymbol{w}} W H S\right)+\boldsymbol{O}\left(n_{w} n_{h} n_{s} H S\right)+ \\
& \boldsymbol{O}\left(n_{w} n_{h} n_{s} W S\right)+\boldsymbol{O}\left(n_{w} n_{h} n_{s} W H\right)+ \\
& \boldsymbol{O}\left(n_{h} n_{s} W H S\right)+\boldsymbol{O}\left(n_{w}^{2} W\right)+\boldsymbol{O}\left(n_{h}^{2} H\right)+ \\
& \boldsymbol{O}\left(n_{s}^{2} S\right)+\boldsymbol{O}\left(n_{w} n_{h}^{2} n_{s}^{2}\right)+\boldsymbol{O}\left(n_{w}^{2} n_{h} n_{s}\right)
\end{aligned}
$$

Noteworthy, the complexity of the proposed method outperforms the other state-of-the-art methods [3, 8]. Unlike the CSTF method [8] and SSSR method [3], As in (36), It is observed that the complexity of the proposed algorithm is quite linear with the size of HSI cube $(W, H, S)$, which is the same as that of conventional NMF algorithm. Owing to the fact that

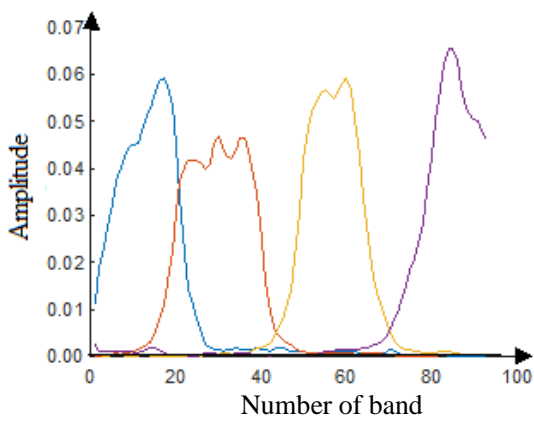

(a) each update step of the proposed CNTD method can be considered as an NMF problem.

\section{EXPERIMENTS AND RESULTS}

\section{A. Data sets}

The proposed CNTD-based method is performed on two wellknown data sets. The first data set is the Pavia University image [45], which is acquired by the reflective optics system imaging spectrometer (ROSIS) optical sensor upon the urban area of the University of Pavia, Italy. The reference HR-HSI of size $120 \times$ $120 \times 93$ with spatial resolution $1.3 \mathrm{~m}$ per pixel and water absorption bands (with a spectral range from 0.43 to $0.86 \mathrm{~m}$ ) removal is illustrated in Fig. 2. The LR-HSI is produced by applying a Gaussian blurring filter and down-sampling it by a factor of 4 . Therefore, the LR-HSI size is $30 \times 30 \times 93$, which is shown in Fig. 2. The HR-MSI with the size of $120 \times 120 \times$ 4 is constructed using the IKONOS like reflectance spectral response function depicted in Fig. 3. (for more details about spectral response and spatial blurring functions see [44]).

The second data set is the Indian Pines image, which was captured by NASA Airborne Visible and Infrared Imaging Spectrometer (AVIRIS) [46]. The reference image is of size $120 \times 120 \times 224$ across the spectral range from 0.4 to $2.5 \mu \mathrm{m}$ with a spatial resolution of $20 \mathrm{~m}$ per pixel. The water absorption and very low SNR bands (1-4, 104-115, 150-170, 223, and 224) are removed and the LR-HSI of size $30 \times 30 \times 185$ is constructed after down-sampling and blurring operation, which is performed just the same as for the Pavia data set. The HRMSI of size $120 \times 120 \times 6$ is produced using the LANDSATlike spectral responses function, depicted in Fig.3.

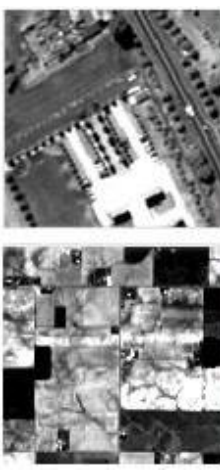

(a)

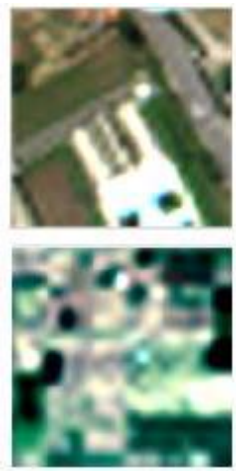

(b)

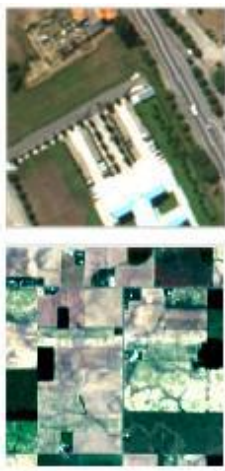

(c)
Fig. 2. The first and second rows show the composite color images of Pavia University and Indian Pines data sets, respectively; (a) HR-MSI, (b) LR-HSI (c) reference image.

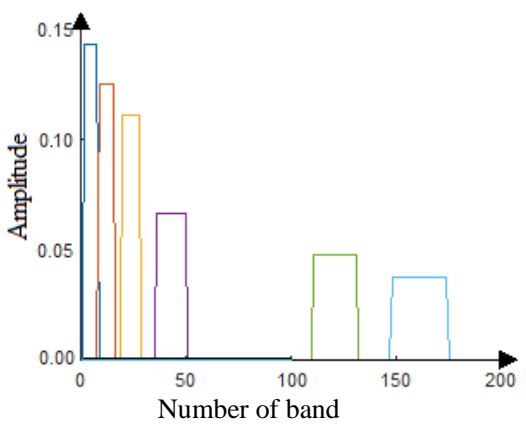

(b)

Fig. 3. Spectral response functions; (a) IKONOS like spectral response function, (b) LANDSAT-like spectral response function 


\section{B. Evaluation criteria}

The performance of the proposed method is validated using the five indexes given below. The first index is the spectral angle mapper (SAM) which measures the spectral distortion between estimated HR-HSI $(\widehat{\boldsymbol{Z}})$ and reference image $(\boldsymbol{Z})$, where $\widehat{\boldsymbol{z}}_{j}$ and $\boldsymbol{z}_{j}$ are pixel spectral responses of $\widehat{\boldsymbol{Z}}$ and $\boldsymbol{Z}$, respectively. It is defined as

$$
\operatorname{SAM}(\boldsymbol{z}, \widehat{\boldsymbol{z}})=\frac{1}{W H} \sum_{j=1}^{W H} \arccos \left(\frac{\widehat{\boldsymbol{z}}_{j}^{T} \widehat{\boldsymbol{z}}}{\left\|\widehat{\boldsymbol{z}}_{j}\right\|_{2}\left\|\boldsymbol{z}_{j}\right\|_{2}}\right)
$$

while the ideal SAM value is zero degree.

The second index is the root mean square error (RMSE) to evaluate the quality of estimated HR-HSI $(\widehat{\boldsymbol{Z}})$ compared with the reference image $(\boldsymbol{Z})$. It is calculated as

$$
\operatorname{RMSE}(\boldsymbol{z}, \widehat{\boldsymbol{z}})=\sqrt{\frac{\|\boldsymbol{z}-\widehat{\boldsymbol{z}}\|_{F}^{2}}{W H S}}
$$

The third evaluation index is error relative globaldimensional synthesis (ERGAS), which also measures the spectral distortion between the estimated HR-HSI $(\widehat{\boldsymbol{Z}})$ and the reference image $(\boldsymbol{Z})$, which is defined as

$$
\text { ERGAS }=100 \frac{W H}{w h} \sqrt{\frac{1}{S} \sum_{i=1}^{S}\left(\frac{\left(\operatorname{RMSE}\left(\hat{\mathbf{Z}}_{i,:}, \mathbf{Z}_{i,:}\right)\right)}{\mu_{\mathbf{z}_{i,:}}}\right)^{2}}
$$

where $\hat{\mathbf{Z}}_{i, \text { : }}$ and $\mathbf{Z}_{i, \text { : }}$ are the $i^{\text {th }}$ bands of $\widehat{\boldsymbol{Z}}$ and $\boldsymbol{Z}$, respectively. $\mu_{\mathbf{z}_{i,}}$ is the mean of $\mathbf{Z}_{i, .}$. In the case of perfect reconstruction, the ideal value of ERGAS is zero.

The fourth index is the degree of the distortion (DD), defined as

$$
\operatorname{DD}(\boldsymbol{z}, \widehat{\boldsymbol{z}})=\frac{1}{W H S}\|\operatorname{vec}(\boldsymbol{Z})-\operatorname{vec}(\widehat{\boldsymbol{Z}})\|_{1}
$$

where $\|\cdot\|_{1}$ is $\ell_{1}$ norm, $\operatorname{vec}(\boldsymbol{Z})$ and $\operatorname{vec}(\widehat{\boldsymbol{Z}})$ are vectorization of tensors $\boldsymbol{Z}$ and $\widehat{\boldsymbol{Z}}$, respectively. Note that the smaller DD, the better spectral quality.

The fifth index is the universal image quality index (UIQI) [47]. It is calculated for windows of size $32 \times 32$ and averaged

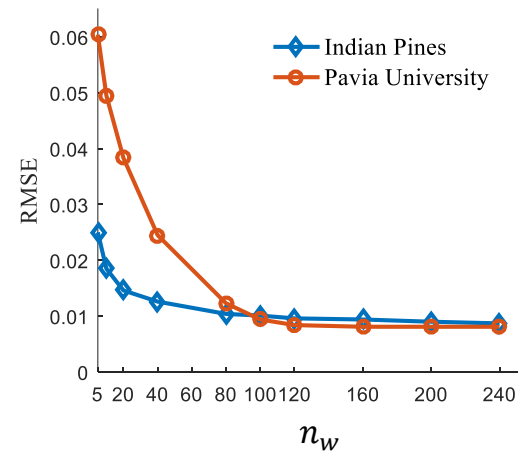

(a)

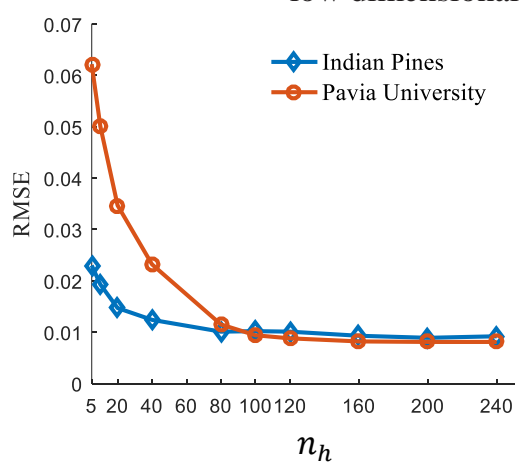

(b) over all windows. The UIQI between the $i^{\text {th }}$ band of $\widehat{\boldsymbol{Z}}$ and $\boldsymbol{Z}$ is given by

$$
\mathrm{UIQI}\left(\mathbf{z}_{i}, \hat{\mathbf{z}}_{i}\right)=\frac{1}{d} \sum_{j=1}^{d} \frac{\sigma_{\mathbf{z}_{j}^{i} \mathbf{z}_{j}^{i}}}{\sigma_{\mathbf{z}_{j}^{i}} \sigma_{\mathbf{z}_{j}^{i}}} \frac{2 \mu_{\mathbf{z}_{j}^{i}} \mu_{\hat{\mathbf{z}}_{j}^{i}}}{\mu_{\mathbf{z}_{j}^{i}}+\mu_{\mathbf{z}_{j}^{i}}} \frac{2 \sigma_{\mathbf{z}_{j}^{i}} \sigma_{\hat{\mathbf{z}}_{j}^{i}}}{\sigma_{\mathbf{z}_{j}^{i}}+\sigma_{\hat{\mathbf{z}}_{j}^{i}}}
$$

where $d$ is the number of windows, $\hat{\mathbf{Z}}_{j}^{i}$ and $\mathbf{Z}_{j}^{i}$ are the $j^{\text {th }}$ window of $i^{\text {th }}$ band of the reference image and reconstructed HR-HSI, respectively, $\sigma_{\mathbf{z}_{j}^{i} \hat{\mathbf{z}}_{j}^{i}}$ is the sample covariance between $\mathbf{Z}_{j}^{i}$ and $\widehat{\mathbf{Z}}_{j}^{i}$, $\mu_{\mathbf{z}_{j}^{i}}$ and $\sigma_{\mathbf{z}_{j}^{i}}$ are the mean and standard deviation of $\mathbf{Z}_{j}^{i}$, respectively. After averaging over all bands, the UIQI index between $\widehat{Z}$ and $\boldsymbol{Z}$ is expressed as

$$
\operatorname{UIQI}(\boldsymbol{z}, \widehat{z})=\frac{1}{S} \sum_{i=1}^{S} \operatorname{UIQI}\left(\mathbf{z}_{i,:}, \hat{\mathbf{Z}}_{i,:}\right)
$$

The UIQI ideal value is one. All the experiments with this subscene are implemented using MATLAB R2016a version run by an Intel Core I5 at $3.4 \mathrm{GHz}$ and $32-\mathrm{GB}$ random access memory computer.

\section{Parameters discussions}

In order to evaluate the sensitivity of the proposed CNTDbased method w. r. t. its essential parameters including the number of mode (width, height, and depth) dictionary atoms $n_{w}, n_{h}$ and $n_{s}$. We run the proposed method for the different number of mode dictionary atoms. Fig. 4 (a), Fig. 4 (b), and Fig. 4 (c) show the RMSE of the estimated Pavia University and Indian Pines data sets as functions of the number of mode dictionary atoms $n_{w}, n_{h}$ and $n_{s}$, respectively. As can be seen from Fig. 4 (a) and (b), the RMSE for both data sets has a steep fall when $n_{w}$ and $n_{h}$ vary from 5 to 200 . However, when they grow higher, the RMSE does not change obviously. Therefore, the $n_{w}$ and $n_{h}$ are set as 167 for both data sets. As Fig. 4 (c) shows, the RMSE for Pavia University decreases as $n_{s}$ varies form 5 to 40. For Indian Pines, the RMSE curve decreases as $n_{s}$ varies form 5 to 100 , and then they do not change obviously as $n_{s}$ increases further. Hence, we set $n_{s}$ as 100 for both Pavia University and Indian Pines data sets, although it gives acceptable results for much lower values of $n_{s}$. Consequently, the proposed CNTD method needs the larger width and height mode dictionaries, and a smaller spectral mode dictionary. The reason is that the spectral signatures of HSIs generally live in low dimensional subspaces.

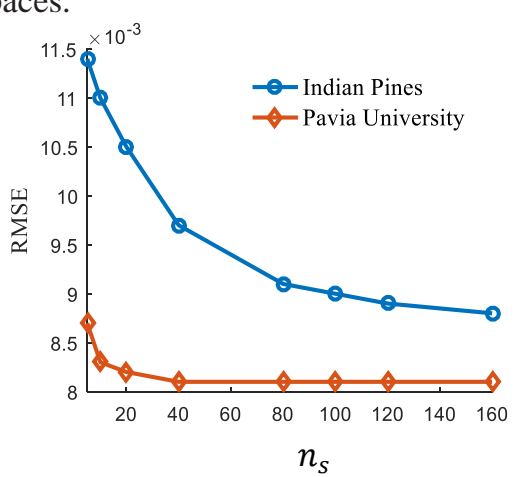

(c)

Fig. 4. The RMSE as functions of the number of atoms $n_{w}, n_{h}$ and $n_{s}$ for the proposed CNTD method; (a) $n_{w}$, (b) $n_{h}$, (c) $n_{s}$. 


\section{Comparison with the other fusion methods}

The proposed fusion method is compared with state-of-the-art methods, which are shown in Table III and IV for Pavia University and Indian Pines data sets, respectively. The comparisons include RMSE, SAM, DD, ERGAS, UIQI, for all approaches. It can be seen that the proposed method outperforms the other competing ones in terms of RMSE DD, and UIQI indexes and promising results for the other indexes. Noteworthy, as can be seen from (36), the proposed method achieves lower complexity order than some of the state-of-theart methods such as [8] and [3] which have non-linear complexity order with the size of HSIs cube.

In order to compare the performance of the competing methods in preserving spatial structures, the error images which reflect the differences between the estimated HR-HSI and reference image for the $30^{\text {th }}$ band of both data sets are shown in Fig. 5. It shows the error images of the LR-HSI, CSTF [8], NLSTF [27] and proposed CNTD method. It can be seen that the proposed method can estimate spatial details of the HR-HSI with much lower error than the others for both data sets.
TABLE III

Quantitative Metrics of the Different Fusion Methods on the PAVIA UNIVERSITY DATA SET [45]

\begin{tabular}{cccccc}
\hline \multirow{2}{*}{ Method } & \multicolumn{5}{c}{ Pavia University data set } \\
\cline { 2 - 6 } & RMSE & SAM & DD & ERGAS & UIQI \\
\hline Ideal value & 0.000 & 0.000 & 0.000 & 0.000 & 1.000 \\
CNMF[25] & 0.011 & 2.039 & 0.009 & 1.089 & 0.985 \\
CSTF[8] & 2.160 & 2.390 & 1.055 & 1.230 & 0.991 \\
CNN [48] & $/$ & 2.230 & 1.370 & 1.338 & 0.992 \\
NLSTF[27] & 1.452 & $\mathbf{0 . 9 6 4}$ & 0.846 & $\mathbf{0 . 5 2 0}$ & 0.993 \\
CNTD method & $\mathbf{0 . 0 0 8}$ & 1.963 & $\mathbf{0 . 0 0 5}$ & 1.169 & $\mathbf{0 . 9 9 6}$ \\
\hline
\end{tabular}

TABLE IV

Quantitative Metrics of THE DifFERENT Fusion Methods on the INDIAN PINES DATA SET [46]

\begin{tabular}{cccccc}
\hline \multirow{2}{*}{ Method } & \multicolumn{5}{c}{ Indian Pines data set } \\
\cline { 2 - 6 } & RMSE & SAM & DD & ERGAS & UIQI \\
\hline Ideal value & 0.000 & 0.000 & 0.000 & 0.000 & 1.000 \\
CNMF[25] & 0.010 & 1.740 & 1.280 & 0.804 & $\mathbf{0 . 9 9 3}$ \\
CSTF[8] & 1.533 & 1.363 & 0.997 & 1.082 & 0.974 \\
CNN [48] & $/$ & 2.270 & 2.090 & 1.060 & 0.820 \\
NLSTF[27] & 0.899 & $\mathbf{0 . 7 6 8}$ & 0.484 & $\mathbf{0 . 7 5 5}$ & 0.984 \\
CNTD method & $\mathbf{0 . 0 0 9}$ & 1.661 & $\mathbf{0 . 0 0 6}$ & 1.249 & 0.972 \\
\hline
\end{tabular}

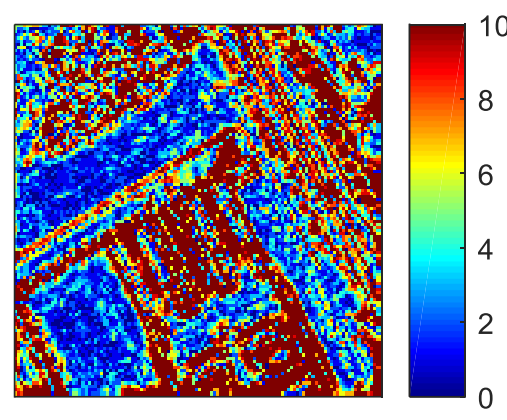

(a)

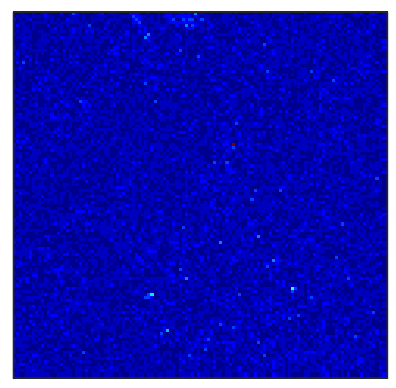

(d)

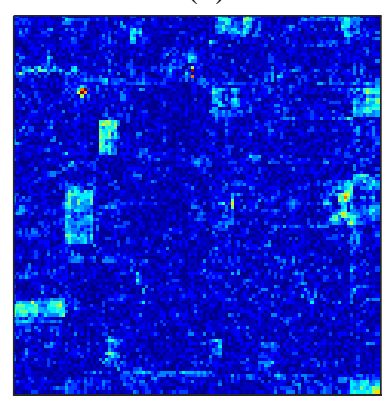

(g)

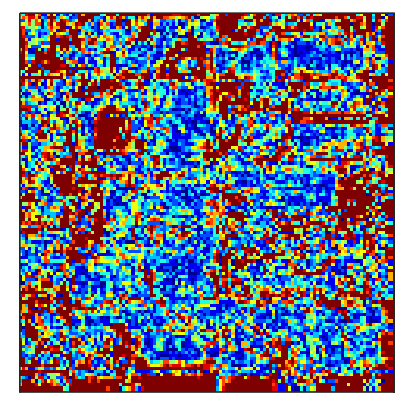

(b)
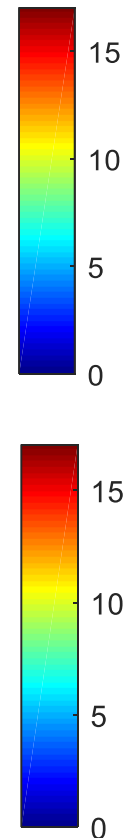

15

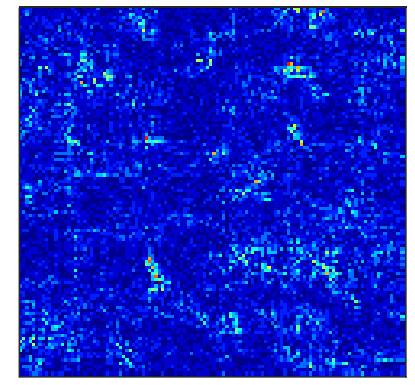

(h)
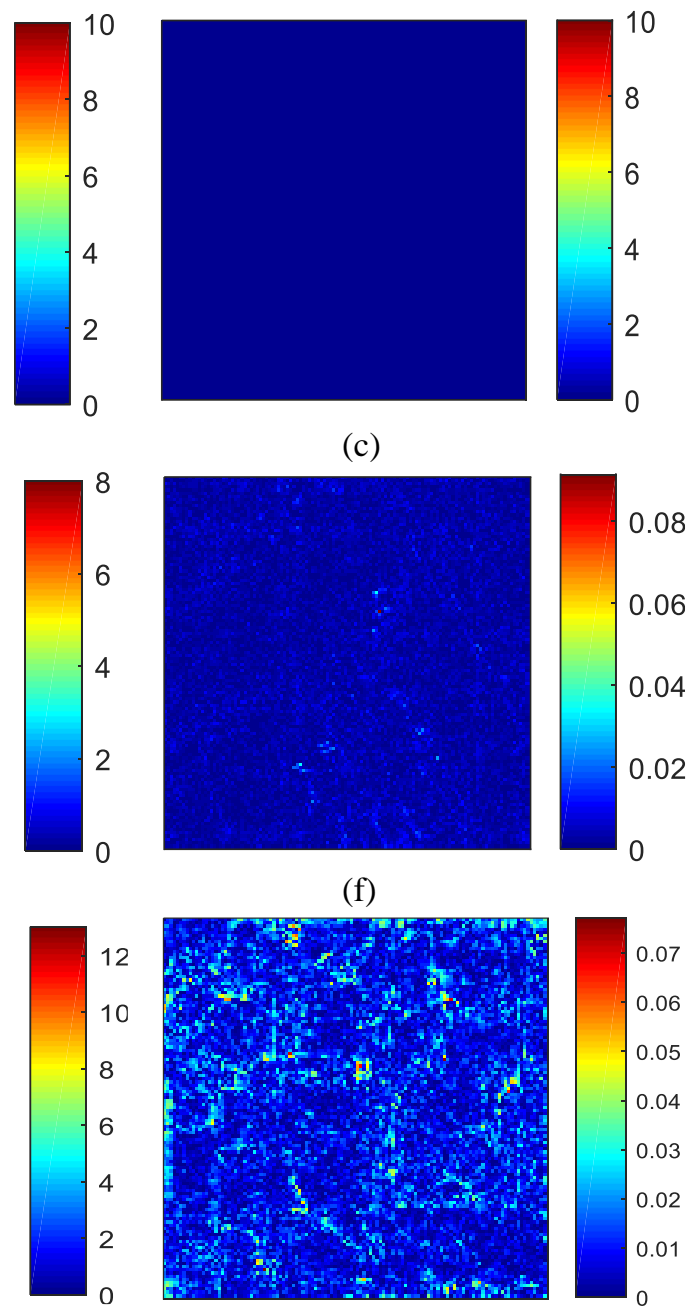

(i)

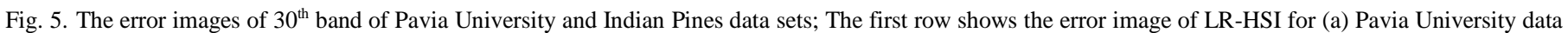

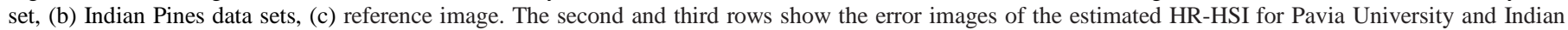
Pines data sets, respectively; (d) and (g) CSTF method [8], (e) and (h) the NLSTF method [27], (f) and (i) proposed CNTD method. 

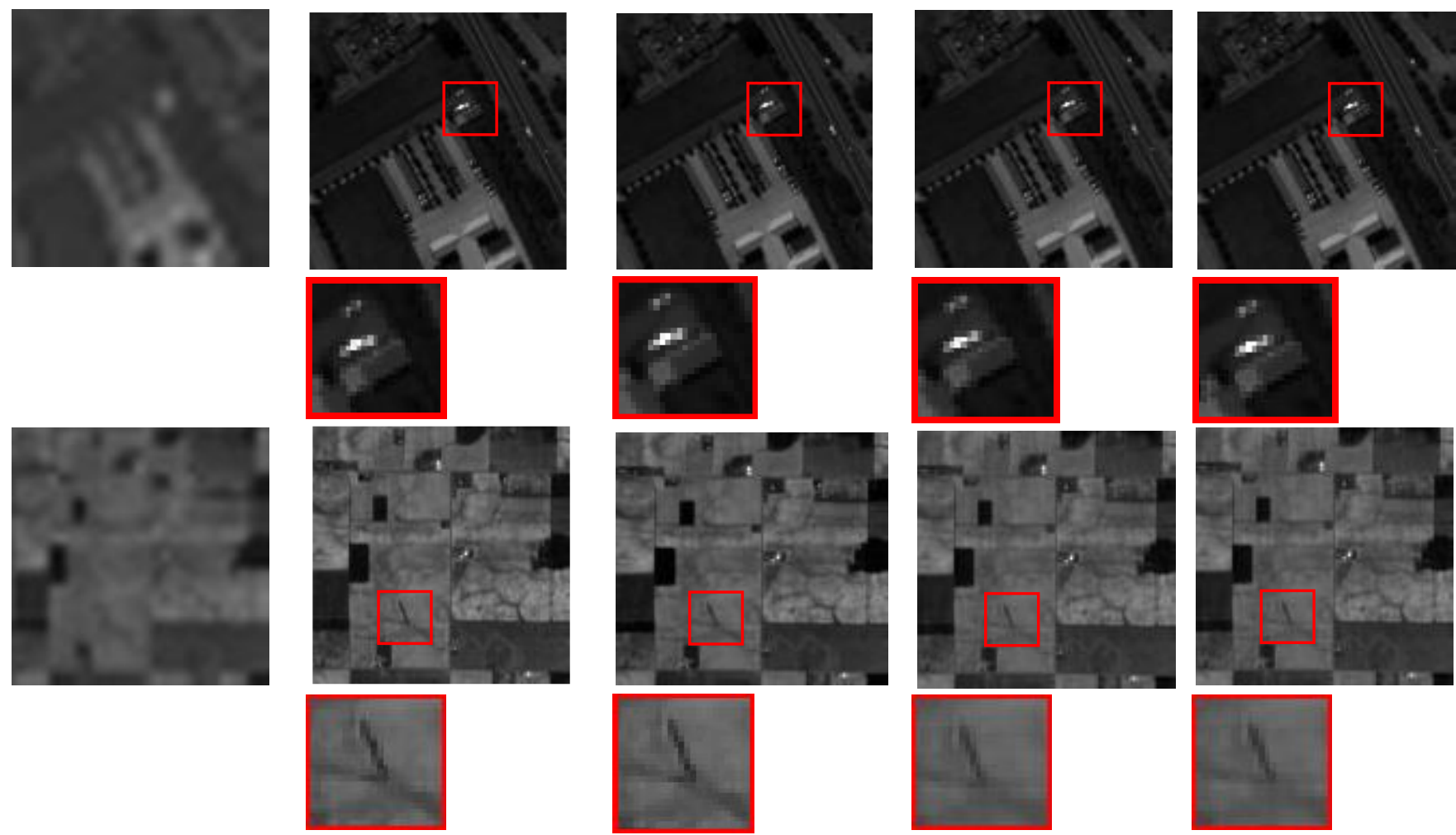

(a)

(b)

(c)

(d)

(e)

Fig. 6. The first and second rows contain the error images of $30^{\text {th }}$ bands of Pavia University and Indian Pines data sets, respectively; (a) LR-HSI, (b) the CSTF method [8], (c) the NLSTF method [27], (d) the proposed CNTD method, (e) reference image.

For more visual comparison the $30^{\text {th }}$ band of LR-HSI and estimated HR-HSI of the CSTF [8], NLSTF [27] and proposed CNTD method are compared with the reference HR-HSI in Fig. 6. It shows that the proposed CNTD method can correctly estimate most of the spatial details of the HR-HSI, though there are a few distortions in the fusion results.

\section{CONCLUSION}

The main object of this paper is to extend NMF to a tensor frame and perform it on LR-HSI and HR-MSI to estimate the super-resolution image. The introduced basic NTD model should be treated as/emphasized like /the conventional NMFbased model, which is quite general in nature with no spatiospectral constraints. According to this, the proposed method is called blind hyperspectral and multispectral Images Fusion using CNTD. The proposed CNTD-based method is compared with the state-of-the-art methods, which gives promising results with quite linear complexity order with respect to the size of the HSIs cube.

As our future work, we can incorporate some prior information, such as spectral self-similarity, sparsity, smoothness, and local consistence besides the non-negative tensor decomposition, they may help to find better unique basis vectors in a Tucker representation.

\section{REFERENCE}

R. Dian, L. Fang, and S. Li, "Hyperspectral image super-resolution via non-local sparse tensor factorization," in Proceedings of the
IEEE Conference on Computer Vision and Pattern Recognition, 2017, pp. 5344-5353.

[2] R. Dian, S. Li, L. Fang, and J. Bioucas-Dias, "Hyperspectral Image Super-Resolution via Local Low-Rank and Sparse Representations," in IGARSS 2018-2018 IEEE International Geoscience and Remote Sensing Symposium, 2018, pp. 4003-4006.

[3] R. Dian, S. Li, L. Fang, and Q. Wei, "Multispectral and hyperspectral image fusion with spatial-spectral sparse representation," Information Fusion, vol. 49, pp. 262-270, 2019.

[4] W. Dong, F. Fu, G. Shi, X. Cao, J. Wu, G. Li, et al., "Hyperspectral image super-resolution via non-negative structured sparse representation," IEEE Transactions on Image Processing, vol. 25, pp. 2337-2352, 2016.

[5] E. Ghasrodashti, A. Karami, R. Heylen, and P. Scheunders, "Spatial resolution enhancement of hyperspectral images using spectral unmixing and bayesian sparse representation," Remote Sensing, vol. 9, p. 541, 2017.

[6] M. V. Joshi and K. P. Upla, Multi-resolution Image Fusion in Remote Sensing: Cambridge University Press, 2019.

[7] C. Lanaras, E. Baltsavias, and K. Schindler, "Hyperspectral superresolution with spectral unmixing constraints," Remote Sensing, vol. 9, p. 1196, 2017.

[8] S. Li, R. Dian, L. Fang, and J. M. Bioucas-Dias, "Fusing hyperspectral and multispectral images via coupled sparse tensor factorization," IEEE Transactions on Image Processing, vol. 27, pp. 4118-4130, 2018.

[9] X. Li, L. Tian, X. Zhao, and X. Chen, "A super resolution approach for spectral unmixing of remote sensing images," International journal of remote sensing, vol. 32, pp. 6091-6107, 2011.

[10] K. P. U. Manunath V. Joshi, Multi-resolution image fusion in remote sensing: Cambridge university, 2019.

[11] N. D. Q. Wei J. Bioucas-Dias, and J. Y. Tourneret, "Hyperspectral and multispectral image fusion based on a sparse representation," IEEE Trans. Geosci. Remote Sens, vol. 53 pp. 3658-3668, 2015. Q. Wei, J. Bioucas-Dias, N. Dobigeon, J.-Y. Tourneret, M. Chen, and S. Godsill, "Multiband image fusion based on spectral 
unmixing," IEEE Transactions on Geoscience and Remote Sensing, vol. 54, pp. 7236-7249, 2016.

[13] J. Yang, Y. Li, J. Chan, and Q. Shen, "Image fusion for spatial enhancement of hyperspectral image via pixel group based nonlocal sparse representation," Remote Sensing, vol. 9, p. 53, 2017.

[14] N. Akhtar, F. Shafait, and A. Mian, "Bayesian sparse representation for hyperspectral image super resolution," in Proceedings of the IEEE Conference on Computer Vision and Pattern Recognition, 2015, pp. 3631-3640.

[15] L. Fang, H. Zhuo, and S. Li, "Super-resolution of hyperspectral image via superpixel-based sparse representation," Neurocomputing, vol. 273, pp. 171-177, 2018.

[16] R. C. Hardie, M. T. Eismann, and G. L. Wilson, "MAP estimation for hyperspectral image resolution enhancement using an auxiliary sensor," IEEE Transactions on Image Processing, vol. 13, pp. 11741184, 2004.

[17] Z. H. Nezhad, Karami, A., Heylen, R., \& Scheunders, P., "Fusion of hyperspectral and multispectral images using spectral unmixing and sparse coding," IEEE Journal of Selected Topics in Applied Earth Observations and Remote Sensing, vol. 9, pp. 2377-2389, 2016.

[18] M. Simoes, J. Bioucas-Dias, L. B. Almeida, and J. Chanussot, "A convex formulation for hyperspectral image superresolution via subspace-based regularization," IEEE Transactions on Geoscience and Remote Sensing, vol. 53, pp. 3373-3388, 2014.

[19] Q. Wei, N. Dobigeon, and J.-Y. Tourneret, "Bayesian fusion of multi-band images," IEEE Journal of Selected Topics in Signal Processing, vol. 9, pp. 1117-1127, 2015.

[20] X. Liu, W. Xia, B. Wang, and L. Zhang, "An approach based on constrained nonnegative matrix factorization to unmix hyperspectral data," IEEE Transactions on Geoscience and Remote Sensing, vol. 49, pp. 757-772, 2010.

[21] X. Lu, H. Wu, Y. Yuan, P. Yan, and X. Li, "Manifold regularized sparse NMF for hyperspectral unmixing," IEEE Transactions on Geoscience and Remote Sensing, vol. 51, pp. 2815-2826, 2012.

[22] Y. Qian, S. Jia, J. Zhou, and A. Robles-Kelly, "L1/2 Sparsity constrained nonnegative matrix factorization for hyperspectral unmixing," in 2010 International Conference on Digital Image Computing: Techniques and Applications, 2010, pp. 447-453.

[23] R. Rajabi and H. Ghassemian, "Spectral unmixing of hyperspectral imagery using multilayer NMF," IEEE Geoscience and Remote Sensing Letters, vol. 12, pp. 38-42, 2014

[24] Z. Yang, G. Zhou, S. Xie, S. Ding, J.-M. Yang, and J. Zhang, "Blind spectral unmixing based on sparse nonnegative matrix factorization," IEEE Transactions on Image Processing, vol. 20, pp. 1112-1125, 2010.

[25] N. Yokoya, T. Yairi, and A. Iwasaki, "Coupled nonnegative matrix factorization unmixing for hyperspectral and multispectral data fusion," IEEE Transactions on Geoscience and Remote Sensing, vol. 50, pp. 528-537, 2012.

[26] R. Dian, S. Li, and L. Fang, "Learning a low tensor-train rank representation for hyperspectral image super-resolution," IEEE transactions on neural networks and learning systems, vol. 30, pp. 2672-2683, 2019.

[27] R. Dian, S. Li, L. Fang, T. Lu, and J. M. Bioucas-Dias, "Nonlocal sparse tensor factorization for semiblind hyperspectral and multispectral image fusion," IEEE Transactions on Cybernetics, 2019.

[28] Y. Xu, Z. Wu, J. Chanussot, P. Comon, and Z. Wei, "Nonlocal coupled tensor cp decomposition for hyperspectral and multispectral image fusion," IEEE Transactions on Geoscience and Remote Sensing, vol. 58, pp. 348-362, 2019.

[29] G. Zhang, X. Fu, K. Huang, and J. Wang, "Hyperspectral SuperResolution: A Coupled Nonnegative Block-Term Tensor Decomposition Approach," in 2019 IEEE 8th International Workshop on Computational Advances in Multi-Sensor Adaptive Processing (CAMSAP), 2019, pp. 470-474

[30] M. Zare, M. S. Helfroush, and K. Kazemi, "Fusing hyperspectral and multispectral images using smooth graph signal modelling," International Journal of Remote Sensing, vol. 41, pp. 8610-8630, 2020.

[31] L. R. Tucker, "Some mathematical notes on three-mode factor analysis," Psychometrika, vol. 31, pp. 279-311, 1966. imagery," IEEE Transactions on Geoscience and Remote Sensing, vol. 55, pp. 1776-1792, 2017.

X.-R. Feng, H.-C. Li, J. Li, Q. Du, A. Plaza, and W. J. Emery, "Hyperspectral unmixing using sparsity-constrained deep nonnegative matrix factorization with total variation," IEEE Transactions on Geoscience and Remote Sensing, vol. 56, pp. 62456257,2018

[34] J. Luan and Z. Zhang, "Prediction of Multidimensional Spatial Variation Data via Bayesian Tensor Completion," IEEE Transactions on Computer-Aided Design of Integrated Circuits and Systems, vol. 39, pp. 547-551, 2019.

[35] M. Marquez, H. Rueda-Chacon, and H. Arguello, "Compressive spectral light field image reconstruction via online tensor representation," IEEE Transactions on Image Processing, vol. 29, pp. 3558-3568, 2020.

[36] B. Yaman, S. Weingärtner, N. Kargas, N. D. Sidiropoulos, and M. Akçakaya, "Low-Rank Tensor Models for Improved Multidimensional MRI: Application to Dynamic Cardiac \$ T_1 \$ Mapping," IEEE transactions on computational imaging, vol. 6, pp. 194-207, 2019.

[37] M. Jouni, M. Dalla Mura, and P. Comon, "Hyperspectral Image Classification Based on Mathematical Morphology and Using Tensor CP Decomposition," Mathematical Morphology-Theory and Applications, vol. 1, 2019.

[38] C. I. Kanatsoulis, X. Fu, N. D. Sidiropoulos, and W.-K. Ma, "Hyperspectral super-resolution: Combining low rank tensor and matrix structure," in 2018 25th IEEE International Conference on Image Processing (ICIP), 2018, pp. 3318-3322.

[39] A. Cichocki, D. Mandic, L. De Lathauwer, G. Zhou, Q. Zhao, C. Caiafa, et al., "Tensor decompositions for signal processing applications: From two-way to multiway component analysis," IEEE signal processing magazine, vol. 32, pp. 145-163, 2015.

[40] A. Cichocki, R. Zdunek, A. H. Phan, and S.-i. Amari, Nonnegative matrix and tensor factorizations: applications to exploratory multiway data analysis and blind source separation: John Wiley \& Sons, 2009.

[41] G. Zhou and A. Cichocki, "Fast and unique Tucker decompositions via multiway blind source separation," Bulletin of the Polish Academy of Sciences. Technical Sciences, vol. 60, pp. 389-405, 2012.

[42] Y.-D. Kim and S. Choi, "Nonnegative tucker decomposition," in 2007 IEEE Conference on Computer Vision and Pattern Recognition, 2007, pp. 1-8

[43] D. D. Lee and H. S. Seung, "Learning the parts of objects by nonnegative matrix factorization," Nature, vol. 401, pp. 788-791, 1999.

[44] M. Simões, J. Bioucas-Dias, L. B. Almeida, and J. Chanussot, "A convex formulation for hyperspectral image superresolution via subspace-based regularization," IEEE Transactions on Geoscience and Remote Sensing, vol. 53, pp. 3373-3388, 2014

[45] F. Dell'Acqua, P. Gamba, A. Ferrari, J. A. Palmason, J. A. Benediktsson, and K. Árnason, "Exploiting spectral and spatial information in hyperspectral urban data with high resolution," IEEE Geoscience and Remote Sensing Letters, vol. 1, pp. 322-326, 2004.

[46] R. O. Green, M. L. Eastwood, C. M. Sarture, T. G. Chrien, M. Aronsson, B. J. Chippendale, et al., "Imaging spectroscopy and the airborne visible/infrared imaging spectrometer (AVIRIS)," Remote sensing of environment, vol. 65, pp. 227-248, 1998.

[47] Z. Wang and A. C. Bovik, "A universal image quality index," IEEE signal processing letters, vol. 9, pp. 81-84, 2002.

[48] J. Yang, Y.-Q. Zhao, and J. C.-W. Chan, "Hyperspectral and multispectral image fusion via deep two-branches convolutional neural network," Remote Sensing, vol. 10, p. 800, 2018.

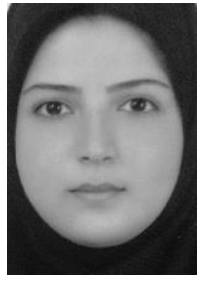

Marzieh Zare received the B.Sc. degree in electrical engineering from Fars University of Science and Research, Marvdasht, Iran, in 2012 and the M.Sc. degree in electrical engineering from Shiraz University of Technology, Shiraz, Iran, in 2015. She is currently working toward the Ph.D. degree in electrical engineering with the Shiraz University of Technology, Shiraz. Her research interests include signal and image processing, image fusion, tensor decomposition and hyperspectral image superresolution. 


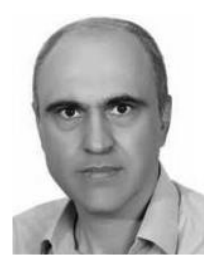

Mohammad Sadegh Helfroush received the B.Sc. degree from Shiraz University, Shiraz, Iran, in 1993, the M.Sc. degree from the Sharif University of Technology, Tehran, Iran, in 1995, and the Ph.D. degree from Tarbiat Modares University, Tehran, in 2006 all in electrical engineering. He is currently a Professor with the Department of Electrical and Electronics Engineering, Shiraz University of Technology, Shiraz. His research interests include different aspects of signal and image processing, pattern recognition, and machine learning.

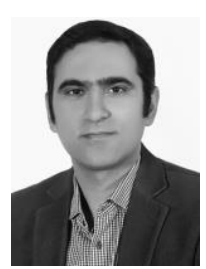

Kamran Kazemi received his B.Sc. and M.Sc. degrees in Electrical engineering from Ferdowsi University of Mashhad and K. N. Toosi University of Technology, Tehran in 2000 and 2002, respectively. He performed his

Ph.D. degree in Electrical Engineering and Biomedical Engineering as cooperation between K. N. Toosi University of Technology, Tehran, Iran and University of Picardie Jules Verne (UPJV) Amiens, France. Currently he is Associate Professor in department of Electrical and Electronics Engineering, Shiraz University of Technology, Shiraz, Iran. His present interests are pattern recognition, image processing and machine learning. 